\title{
Research into the Work Performance of Older Employees
}

\author{
by Peter Warr*
}

\begin{abstract}
There is much simplistic and inaccurate thinking about older workers. That is associated with stereotypes that are incomplete, and a failure to appreciate that no one person (of any age) is well-suited to all possible jobs. These points are developed in this article, which also emphasizes the potential adaptability of employees of all ages.
\end{abstract}

\section{Stereotypes of older workers}

The use of stereotypes is a normal part of thinking. No-one is able to cope with all the information which is available about individual people and objects. Instead, we often think about groups as a whole. For example, it is common to have views about groups such as the French, the English, engineers, accountants, or even older workers.

Stereotypes are not necessarily entirely incorrect. When all the members of a group are similar to each other in the feature in question, a collective judgement about that group might validly apply to its individual members. However, when group members differ widely among themselves, a single summary description is inevitably wrong in many cases. Studies of age patterns have shown that the range of differences between people tends to become even greater in older age-groups; that great variability means that stereotypes about older workers are particularly liable to be wrong when applied to specific individuals.

It is often said that older employees as a whole are viewed more negatively in all respects than are younger ones. In practice, however, stereotypes of the group can have both a negative and a positive component. This can be demonstrated in terms of findings from a survey of members of the British Institute of Personnel Management.

The managers were asked to compare older and younger non-managerial workers at their site on a range of different performance dimensions. "Older workers" were defined as those aged 40 or above. Statistical analyses revealed that there were two clear factors in the judgements. In one factor, older workers were perceived as less effective than younger ones, but in the second case it was the older group which was seen as more effective.

The two factors are summarized in Table 1. In the first case, Adaptability, it is not surprising that older employees were seen as slower at acquiring new skills and knowledge. We know from other research that that is often the case, although there are wide differences in Adaptability at all ages.

\footnotetext{
* Department of Psychology, University of Sheffield, Sheffield S10 2TN, England.
} 
Table 1:

Personnel managers' views of older non-managerial workers at their site

1. Adaptability: Older workers are poorer than younger workers

- Able to grasp new ideas

- Adapt to change

- Accept the introduction of new technology

- Learn quickly

2. General work effectiveness: Older workers are better than younger workers

- Are reliable

- Work hard

- Are effective in their job

- Think before they act

- Have interpersonal skills

- Work well in teams

However, the larger factor was in terms of what is labelled in the table as General Work Effectiveness. That covered a wide variety of behaviours, and in every case the older group was seen as significantly more effective: in terms of reliability, conscientiousness, work effectiveness, carefulness, and interpersonal skills.

As will be described later, other research has shown that this two-part stereotype is more valid than the widely-held assumption that older employees are deficient in all respects. Investigations have shown that older workers are on average more effective than younger ones in many ways (factor one, above), but that in certain key respects they may have difficulties. Those difficulties are likely to be in terms of Adaptability, and they are specially significant in the present period of rapid technological change.

It follows that a sound practical approach is for organizations not to reject older workers on the basis of an excessively pessimistic overall negative stereotype. Instead, they should concentrate on enhancing Adaptability, in order to obtain the widespread benefits of experience which older employees provide. This issue will be taken up again later.

\section{Work performance: Research findings}

The overall finding from more than 100 investigations is that there is no general difference in work effectiveness between older and younger workers. In terms of coefficients of correlation, the average value is around zero, but separate associations with age in different studies range from strongly negative to strongly positive. There are clearly differences in that relationship across jobs and between different dimensions of performance (sometimes older workers are better, sometimes they are worse, and often there is no difference), but the general pattern is clear; there is no overall difference between the performance observed for older and younger staff in the same job. Incidentally, in almost every case variations within a particular age-group far exceed the average difference between age-groups. 
The absence of an overall age effect in work performance contrasts sharply with evidence from psychological experiments. There have been many investigations in the laboratory which consistently demonstrate (quite small) decreases in some forms of informationprocessing effectiveness during the working years.

We clearly need to learn about the factors determining the age-patterns in different situations and in different behaviours. Looking at individual research reports is not very helpful in that respect, because they usually give very little information about the nature of the jobs and the individuals concerned. In addition, results are usually presented only in summary form (for example, a correlation coefficient), so that we cannot look at actual levels of scores for different age-groups. However, six examples are given in Table 2, in respect of actual work output.

Table 2:

Age and job performance: Examples of research findings through measures of output

\begin{tabular}{|c|c|c|c|c|c|}
\hline & $\begin{array}{l}\text { under } \\
25\end{array}$ & $25-34$ & $35-44$ & $45-54$ & $\begin{array}{l}55 \text { and } \\
\text { above }\end{array}$ \\
\hline $\begin{array}{l}\text { 1. Skilled manufacturing } \\
\text { operators (USA) }\end{array}$ & 77 & 85 & 100 & 106 & 106 \\
\hline $\begin{array}{l}\text { 2. Semi-skilled assembly } \\
\text { workers (USA) }\end{array}$ & 89 & 87 & 100 & 105 & 101 \\
\hline 3. Mail sorters (USA) & 101 & 102 & 100 & 101 & 99 \\
\hline 4. Office workers (USA) & 92 & 99 & 100 & 99 & 98 \\
\hline $\begin{array}{l}\text { 5. Manufacturing machine } \\
\text { operators (USA) }\end{array}$ & 96 & 100 & 100 & 97 & 94 \\
\hline $\begin{array}{l}\text { 6. Equipment service } \\
\text { engineers (UK) }\end{array}$ & & 99 & 100 & 94 & \\
\hline
\end{tabular}

Standardizing the scores around those for workers aged between 35 and 44, there is in these studies generally an increase in output up to that age-range, and then either a continuing increase, a plateau, or a small decline. In respect of the equipment service engineers, there is a more rapid decline after the middle range. However, it is important to stress that the decline at older ages in that study was found only for the sub-set of engineers who had not recently been trained.

What about other aspects of work behaviour? In very general terms, the picture is as follows. Although sickness absence is usually found to be more extended at older ages, the opposite is the case for "voluntary" absence, when people take short periods of time off from work without medical or organizational approval. The overall age-pattern of absenteeism 
thus depends on the mix of those two effects in a particular organization, and in many cases there is no overall age difference. Second, accidents are more common at younger ages, especially among inexperienced workers. And, third, older staff are less likely to leave their employer voluntarily, partly of course since they tend to be relatively unattractive in the labour market. One implication of the lower staff turnover at older ages is that the financial pay-back from training older employees can be greater than in the case of younger ones, since younger staff are more likely to move away to another employer.

\section{A four-category framework}

A general theme of this review is that we should not expect to find a universal decline in job performance with increasing age. There are many work activities in which age is a definite advantage or at least neutral. That positive perspective contrasts sharply with the findings from laboratory studies. Those investigations focus upon maximum performance in relatively complex activities, often in novel circumstances where speed of response is specially important. However, work behaviour is not usually of that kind. A pcrson rarely has to operate at maximum levels for more than a short period, many situations are familiar ones requiring well-practised behaviour, and success is typically a function of motivational factors and interpersonal skills as well as merely aspects of cognitive functioning.

A related point concerns the effectiveness of people's overall work strategies. Irrespective of specific mental activities, as assessed in laboratory experiments, people differ in their overall approach to a job, its planning, how they combine different elements together or in sequence, and how they choose which tasks to prioritize and which to treat as less important. Those higher-level processes are sometimes referred to as "metacognitive" activities, concerned with strategies and overarching perspectives, and there is no reason to expect them all to deteriorate with age.

In seeking to identify aspects of jobs that are likely to remain stable or to change across the working years, a four-category framework may be useful, as illustrated in Table 3.

Those four categories are of course overlapping rather than being completely separate. And the key problem is to determine which particular job activities fall into each category. However, the diagram clearly makes the point (in the fourth column) that a negative relationship with age (such that older workers are worse) is expected in only one out of four sets of conditions (Task Category 2).

The diagram is based on the combined effects of two features which are important during ageing. First are basic capacities of a physiological or fundamental psychological kind. For instance, it is well established that response speed and selective attention decline with age, especially in difficult tasks. In those activities (in Task Categories 2 and 3), basic capacities are more exceeded by task demands as people become older. Incidentally, most declines of that kind are likely to be greatest after the age of retirement from the labour force, rather than during the years of paid employment.

However, there are also circumstances in which basic capacities are not further exceeded as people grow older; those are shown as Task Categories 1 and 4 . Working within capacity usually occurs in settings where response speed is of no great consequence, where established routines are being followed, or where the task is quite simple. We should remember that many work activities are of those kinds. 
Table 3:

Four categories of job activity and expected relationships of performance with age

\begin{tabular}{lllll}
\hline Task & Task requirements & Performance can \\
category & $\begin{array}{l}\text { more exceed basic } \\
\text { capacities with } \\
\text { increasing age }\end{array}$ & $\begin{array}{l}\text { be enhanced by } \\
\text { relevant } \\
\text { experience }\end{array}$ & $\begin{array}{l}\text { relationship } \\
\text { with age }\end{array}$ & $\begin{array}{l}\text { Illustrative } \\
\text { job content }\end{array}$ \\
\hline
\end{tabular}

1. Age-enhanced activities

No Yes $\quad$ Positive $\quad$ Knowledge-based judgment with no time pressure

\section{Age-impaired activities}

Yes

No

Negative

Continuous, paced dataprocessing; rapid learning; heavy lifting

\section{Age-counteracted activities}

Yes

Yes Zero

Skilled manual or cognitive work

\section{Age-neutral activities}

No

No

Zero

Relatively undemanding activities

The second factor in this framework (in column three) concerns the gains from experience which come with increasing age. People acquire useful knowledge and skills, routines of behaviour, effort-saving procedures and strategies, and wider perspectives on problems than may be the case at younger ages. However, those gains are of benefit in only some activities, so it is important to consider both possibilities: where performance either is or is not enhanced by experience ("yes" or "no" in column three).

Within Task Category 1 in the framework, work remains within basic capacities despite advancing years and performance improves as a result of experience. Activities in this category may be referred to as age-enhanced. Note, incidentally, that the effect of experience may not be linear, continuing equally across all years in a job; there are grounds for expecting the benefits from experience to level off after an initial period of enhancement.

Examples of Task Category 1 often involve knowledge acquired in the course of a job or a similar position. For instance, in a study of an American company's sales staff older employees were rated very much more positively than younger ones in almost every respect. The correlation between age and an employee's knowledge of the company's products was particularly high and positive. The investigator reported that the association arose from the fact that "since the company carries over 1,000 items in its catalogue, a man may require 
many years to know the technical characteristics of the majority of them". Increased age and time-in-the-job are clearly very helpful in such a setting. Also within this first category are standardized measures of verbal intelligence or vocabulary; they tend to increase slightly with experience across the working years.

A longer period in a particular area of work (which is generally associated with age) brings about mental and behavioural changes associated with increased expertise. There is more "automatization" of actions, as people move from effortful thinking under conscious control to be able to initiate strings of action with little mental effort. Experts in a domain are also more able to perceive chunks of information as a whole, to draw upon previous knowledge, to recognize meaningful patterns, and to hold in mind complex sets of interdependent information.

However, at the opposite extreme (in Task Category 2 of Table 3), basic capacities are exceeded to a greater extent for older people, and experience cannot help. Age-impaired activities of that kind include continuous rapid information-processing and some kinds of strenuous physical activity. It is clear that older people are at a disadvantage here; and this is the situation investigated in most laboratory research.

For example, reaction times to new stimuli are known to be longer at older ages. This slower handling of information may give rise to poorer performance on any task that requires rapid mental processing. Everyone's cognitive capacity is limited, and there is need to pass information through the system as rapidly as possible before it is lost or overtaken by other material. Particularly complex tasks, requiring a larger number of processing steps, are specially likely to be susceptible to this form of slowing.

Among age-impaired activities in Task Category 2 are processes of "working memory". A person often has to carry out mental operations on one set of material, while retaining in temporary storage material which has to be brought into active processing later in the task. Items in working memory are more likely to become forgotten or inaccessible as a person grows older. Since working memory is centrally involved in many complex mental processes, that deterioration can have negative effects on a range of activities.

In the age-counteracted activities of Task Category 3 , older people may have increasing difficulties because of a decline in information-processing or physical capacities, but can counteract that decline in some way. This is the type of activity for which models have been developed in terms of "selective optimization with compensation". Those models are based on the fact that, as people age, they are able to increase their effectiveness in areas of specialization. Continued interest and practice in a limited number of areas permit the growth of knowledge-based expertise ; and individuals are sometimes able to learn how to compensate if limitations arise from deteriorating capacities.

A much-quoted example of this combined effect is a study of typists aged between 19 and 72 . Although older typists were clearly impaired in separate measurements of their response speed, they were able to type as fast as the younger ones. It turned out that older typists achieved this by looking further ahead along the line to be typed, so that they were processing at any one time longer chunks of material than were younger typists. That greater anticipation permitted older people to compensate for declining perceptual-motor speed.

Activities in Task Category 4 are those in which work is unproblematic and fairly routine, such that no noticeable age gradient in either direction is expected. Many work tasks 
are of course of that kind, described in Table 3 as age-neutral. For example, simple learning tasks are unaffected by age, material is remembered equally well providing it has been well learned, expertise continues to be available, everyday decision-making remains effective, and the large majority of individual behaviours and interpersonal routines are carried out without any impact of age.

The published research about job performance is not easily fitted into this four-part framework, since very little information about task activities and sampling procedure is included in most reports. Much of the published research is also rather old, and we now need some new and more complex investigations. My colleagues and I are working with several organizations to try to create a firmer research base, and would be delighted to hear from anyone who might like to collaborate in this work.

\section{Some key issues}

Three key issues now need attention. First, we must look much more carefully at those features of jobs and career progression which might underlie an observed age pattern. There is at present too great a willingness to take an observed correlation with age at its face value, assuming that it reflects a change with increasing years.

For instance, a negative age gradient, where older job-holders are seen as less effective than younger ones, can arise for a range of organizational reasons. In some cases, the best workers will already have been promoted out of the position; or the personnel selection procedures may have become more rigorous in recent years. Comparisons between older and younger job-holders in these situations are not comparing like with like. In cases of selective promotion out of a job, we cannot infer from lower scores observed at older ages that older job-holders are necessarily less effective or that individual people's performance has declined over the years. We can conclude only that the current older incumbents are relatively poor performers, perhaps because of differences in the kinds of people involved at the different ages. Age-differences observed in that setting may thus have nothing directly to do with individual processes of the kind shown in Table 3 ; they result more from promotional policies and other organizational procedures.

In other cases, a positive age gradient (where older employees are seen as more effective) might also arise artefactually. It sometimes happens that an investigator combines within a single analysis several levels of a job, which together form a career progression. In that case, the statistical tendency in supervisors' ratings will be toward a positive age gradient : older people are more often in the higher grade, which is itself perceived more positively. The pattern observed there may be very different from the findings which would be obtained within merely a single grade of job.

A second important issue concerns the bases of between-person variations. The magnitude of any negative age gradient is not the same for all kinds of people, and we need to learn more about the reasons for differences between people in their pace of ageing. If we knew more about that, we could perhaps act to reduce decrements in particular cases.

For example, what about the decline which occurs in basic psychological capacities: how might we explain between-person variations in the steepness of those declines? In respect of cognitive decrements, there is accumulating evidence that differences in cardiovascular fitness may be implicated, associated with oxygen transport to the brain. For example, it has been shown that older employees whose aerobic fitness is at a high level are 
as effective as younger ones in certain forms of information-processing, whereas their older colleagues who are less aerobically fit exhibit the reduced effectiveness which is more usually found. However, although this factor is known to affect information-processing in the laboratory, research of this kind has not yet extended into actual work settings.

Another possible influence on the pace of ageing is the degree of exposure to an intellectually challenging environment. It is possible that workers who are required to operate at a high level of mental complexity throughout their adult life may show less intellectual decline than those who have little cognitive challenge in their jobs; the former, as it were, remain well practised at information-processing tasks.

This has been shown in a limited way through studies of hand-writing speed. Some workers continue writing in their everyday work, whereas others do not: differences in the magnitude of age-decline in hand-writing speed might be expected to parallel the amount of practice acquired across lengthy periods of employment. One study recorded the maximum speed with which people between 40 and 69 could copy a sheet of one-digit numbers. For manual labourers and managers, speed of writing was significantly slower at the older ages, but clerical workers (who performed that kind of task regularly in their job) showed no difference in speed associated with increasing age. It seems possible that this kind of effect will also be demonstrated for more complex mental processes and behaviors; the pace of ageing is not completely fixed in advance for an individual, but is also influenced by his or her environment. Some jobs (and other environments) are more conducive than others to "successful ageing".

A third set of issues concerns the second feature in the framework of Table 3 : performance enhancement by experience. Relevant experience can often be provided by an employing organization, so that in many cases a specific negative age gradient can in fact be prevented or reduced. To put that another way, if we find that older employees are at present less effective than younger ones in a particular job, that difference may not be fixed and unchanging. It could be that the older workers mainly need more opportunities to learn.

In most organizations, training is directed especially at younger employees. This is partly because many employers think they are likely to gain a better financial return from younger people, since they have more years ahead of them. But the emphasis on younger worker's training is also sometimes due to what is in effect collusion between management and older workers themselves. Many older people are nervous about being trained, they feel that they are less able to learn than previously, and they are not sure that they will subsequently have opportunities to use many of the new skills they might acquire.

So there is sometimes a self-fulfilling prophecy: older workers in a particular company may indeed be less Adaptable (factor one in Table 1), but usually they receive no assistance to be otherwise. As a result, they remain as they are, and the negative stereotype is reinforced. However, in view of older employees' clear strengths in respect of General Work Effectiveness (factor two in the table), the major requirement is to create organizational cultures in which learning and development are given a high priority among older as well as younger employees. Companies should assist older workers to move laterally into novel and challenging jobs, and provide training which is specially tailored to their requirements. Despite many negative beliefs, older employees can learn extremely well; but the conventional training techniques devised for younger learners are sometimes inappropriate at older ages. 
Issues of training are thus at the heart of "the problem" of older workers. A negative age gradient in job performance may sometimes be observed, but in many cases that is likely to be at least in part reversible through appropriate training and learning activities. By enhancing Adaptability, employers can also gain from older staff those additional benefits which are known to be present in other forms of General Work Effectiveness. 